\title{
SEM Through Dielectric Membranes: Secondary Electron Contrast Reversal
}

\author{
D.N. Leonard ${ }^{1}$, D. S. Gardiner ${ }^{2}$, and R. L. Thomas ${ }^{2}$ \\ 1. Materials Science \& Technology, Oak Ridge National Laboratory, Oak Ridge, TN USA \\ 2. Protochips, Inc. Raleigh, NC, USA
}

Recent advances in electron microscope fluid cell design have enabled imaging of samples in aqueous environments and have provided a platform to perform in-situ crystallization experiments as well as electrochemical studies [1-2]. However, the actual SEM imaging contrast mechanisms when imaging through the dielectric membranes of this type of fluid cell device remain unknown. In these experiments, high atomic number materials (e.g. Au, Ag) were imaged in the fluid cell using practical SEM imaging parameters and the resulting imaging contrast was documented.

A Protochips Poseidon 200 fluid cell holder, with $\mathrm{SiN}_{\mathrm{X}}$ windows measuring $\sim 40 \mathrm{~nm}$ thick, was employed in both a Hitachi NB5000 FE-FIB/SEM and a Hitachi S4800 FE-SEM. The SiN dielectric $_{\mathrm{x}}$ membranes of the Poseidon 200 created a channel, as illustrated in Figure 1, where static and dynamic fluid cell experiments were carried out. Typical probe currents and scan speeds were used to acquire SE, BSE, and STEM BF/DF images. SEM accelerating voltages between $1 \mathrm{kV}$ and $30 \mathrm{kV}$ were used and sample chamber vacuum remained at 3E-4 Pa throughout the experiments. To simplify interpretation of the resulting imaging contrast, SEM imaging of dry-and deionized (DI)-water-filled channels without samples was performed first. These results were then compared with the contrast from dry- and wetimaging of 40nm Au nanoparticles and Ag flakes present in the fluid channel.

When imaging an empty fluid channel at $4 \mathrm{kV}$, as shown in Figure 2A, bright contrast was produced in the SE image from the channel region. After DI water was flowed into the channel, the SE contrast was reduced due to the introduction of the fluid (Figures 2B,C). Imaging 40nm Au nanoparticles in a dry channel at $4 \mathrm{kV}$ and $25 \mathrm{kV}$ resulted in the observation of a contrast reversal in the SE images (Figure $3 \mathrm{~A}$, $\mathrm{B}$, respectively). The inset of Figure $3 \mathrm{~A}$ shows a cluster of $\mathrm{Au}$ nanoparticles imaged at $4 \mathrm{kV}$, which appears as a dark shadowy feature in the SE image. When the same cluster of Au nanoparticles was imaged at $25 \mathrm{kV}$, the resolved $\mathrm{Au}$ nanoparticles produced bright $\mathrm{SE}$ contrast, as shown in the inset of Figure 3B.

Effects of increasing the electron beam from $1 \mathrm{kV}$ to $25 \mathrm{kV}$ were also studied with a DI-water-filled channel, which contained Dm-sized Ag flakes. At $1 \mathrm{kV}$ (Figure 4A) the electron beam did not pass through the $\mathrm{SiN}_{\mathrm{x}}$ membrane as indicated by no $\mathrm{SE}$ contrast from the Ag flake and predicted by the monte carlo simulation of electron beam interaction volume (inset Figure 4A). At a much higher voltage of $25 \mathrm{kV}$, the morphology of the Ag flake was clearly observed in the SE image (Figure 4D).

These results show that interpreting SEM image contrast from SEM fluid cell experiments can be complicated by the complex beam, sample, and $\mathrm{SiNx}$ membrane interactions [3-4]. Individual contributions from the dielectric membranes, the fluid used, and the sample material, which will be discussed must all be considered before SE, BSE, and STEM BF/DF data from fluid cell experiments can be interpreted.

\section{References:}

[1] N. de Jonge and F.M. Ross, Nature Nanotechnology 6 (2011), pp. 695-704.

[2] R.L. Sacci, et al, Chemical Communications 50 (2014), pp. 2104-2107.

[3] D.J. Stokes, et al, Scanning 22 (2000), pp. 357-365.

[4] D.C. Joy and C.S. Joy, Journal of Microscopy 221 (2006), pp. 84-88. 


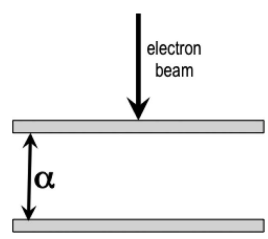

Figure 1. Two SiN membranes integral to the fluid cell device isolate liquid from vacuum of the SEM specimen chamber. The gap width $(\alpha)$ of the channel can be chosen to be as small as $150 \mathrm{~nm}$ or as wide as $5 \mu \mathrm{m}$.
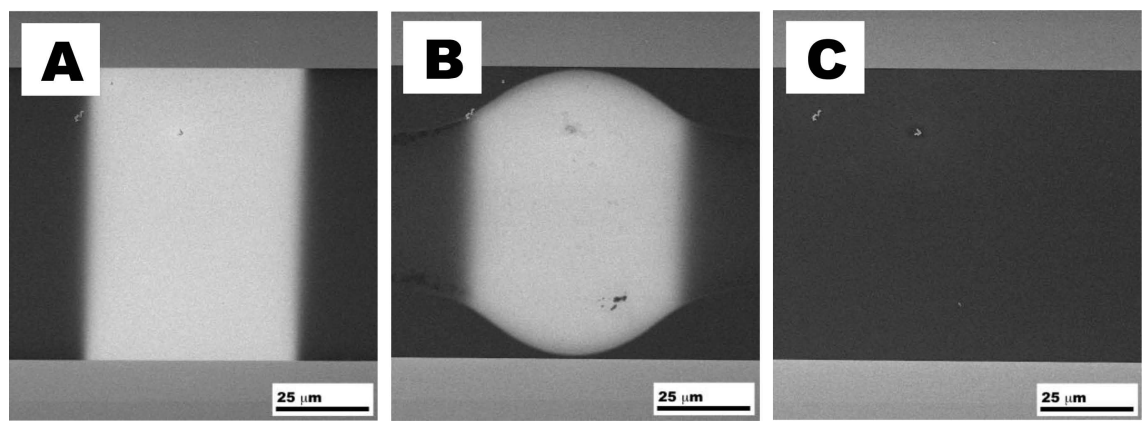

Figure 2. [A] Imaged at $4 \mathrm{kV}$, the dry channel produced a region of bright contrast in the $\mathrm{SE}$ micrograph. [B] Under identical SEM conditions the SE contrast changed in regions where DI water filled the channel. [C] Darker SE contrast resulted when the DI water filled channel was imaged using identical SEM imaging conditions as $[\mathrm{A}]$ and $[\mathrm{B}]$.
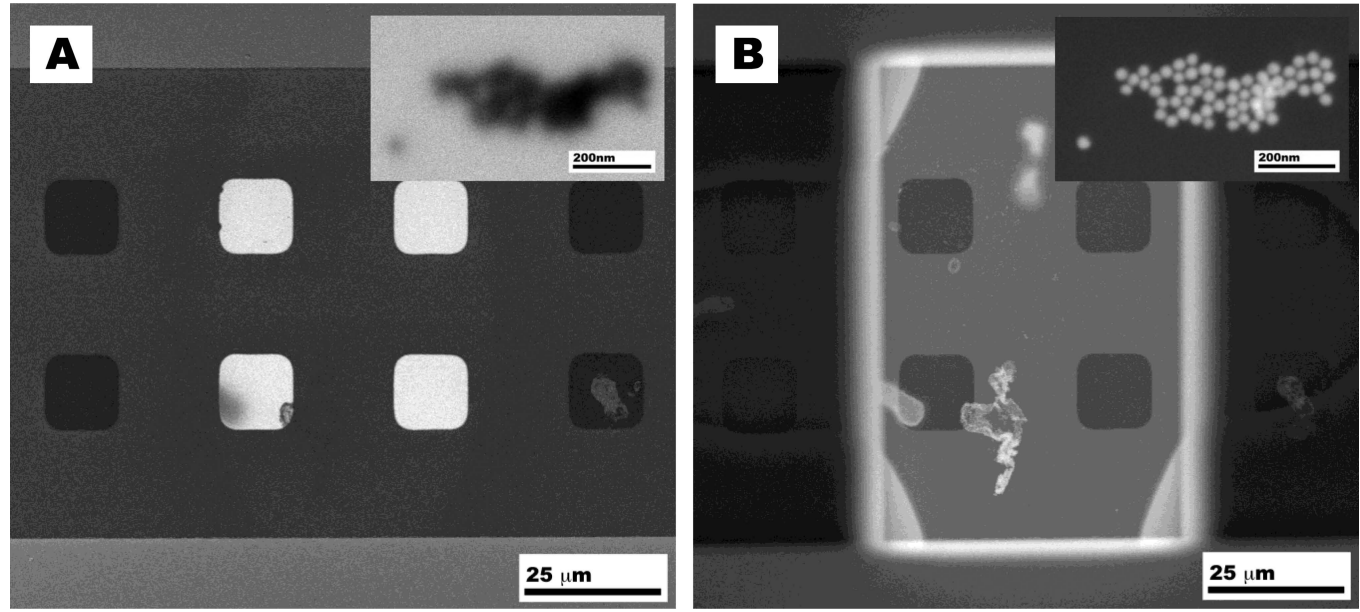

Figure 3. [A] Dry channel containing Au nanoparticles produced poor spatial resolution and dark contrast from a cluster of nanoparticles (inset) when imaged at $4 \mathrm{kV}$. [B] Identical sample, imaged at $25 \mathrm{kV}$, resolved individual $40 \mathrm{~nm}$ Au particles (inset) and resulted in bright contrast from the high atomic number nanoparticle cluster.
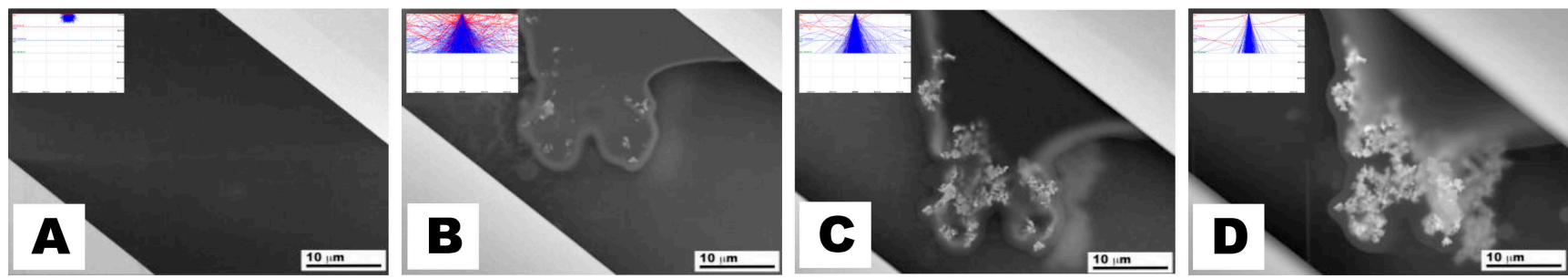

Figure 4. In-lens SE detector was used to acquire micrographs of a silver particle at $[\mathrm{A}] 1 \mathrm{kV}$, [B] $5 \mathrm{kV}$, [C] $10 \mathrm{kV}$ and [D] $25 \mathrm{kV}$. Insets of each image show the monte carlo simulated theoretical electron beam interaction volume at each $\mathrm{kV}$ used to acquire the micrograph. 